\title{
Understanding the Possible Links Between Grain Boundary Morphology and Mineralogy in the Sericho Pallasite
}

\author{
Shawn Wallace ${ }^{1}$ and Jens Rafaelsen ${ }^{1}$ \\ 1. EDAX, Ametek, Mahwah, NJ, USA.
}

Pallasites are type of stony-iron meteorites that contain large olivine grains surrounded by an iron-nickel metal matrix. This mineralogy denotes differentiation of the parent body. Besides this basic differentiation, most hypothesis of formation have different ways of explaining the mixed nature of the morphology of the olivines and relationships between the different minerals present. Most hypotheses start with basic fractional crystallization, but then branch in to other modes of differentiation/convection of materials [1], or invoke impact related events [2]. The main observations that these two hypotheses have to consider is the coexisting nature of rounded, euhedral, and fragmented olivine grains, often in the same meteorite, while surrounded by metal matrix. This study looks at the morphology of the olivine grains, while discussing possible mineralogical control.

A polished slice of Sericho $(\sim 24 \mathrm{x} 40 \mathrm{~mm})$ was the basis of the study. The sample was carbon coated and mounted in a JEOL-7000F FEG SEM. EDS mapping data was collected on an EDAX Octane Elect. Maps were collected at $15 \mathrm{k}$ counts per second with an accelerating voltage of $15 \mathrm{kv}$. Each map consisted of 64 frames, with 200 usec dwell time per pixel, with resolutions of 512x400 pixels.

Only two articles have been published on Sericho so far, with one being the initial classification [3] and another focusing on the chemistry of chromites [4]. Both note the presence of troilite and schreiberstite, while [4] notes the absence of any phosphates. Olivine grains that exhibit both rounded and euhedral faces are described.

EDS maps of the sample showed abundant evidence for the presence of phosphates. Closer examination showed these phosphates to be the minerals merrillite and stanfieldite. These minerals were sometimes found present next to each other (figure 1a) or alone (figure 1b). Stanfieldite was also found between olivine grains, with the grain boundaries being straight (figure $1 \mathrm{~b}$ and figure 2), while other faces of the same olivine crystal show rounding behaviour next to sulfides and metals (figure 2.) Figure 2 shows clear triple junctions between the phosphates and 2 olivine grains. No farringtonite was found.

The clear difference in the grain boundary morphology shows that the interfacial energy of the metal to silicate phases have a control over said morphology. Contrast this with the phosphate/silicate and silicate/silicate grain boundaries, which show a more annealed texture. These morphologies could be due to fractional crystallization, specifically following the route noted in [2], stopping before farringtonite would form. The combinations of these rounded vs. angular grain boundaries could be interpreted as either crystallization, recrystallization, or even resorption.

Evidence of which of these process (crystallization, recrystallization, or resorption) occurred on these grains could be apparent in maps derived from Electron Backscatter Diffraction that look at orientation deviations within individual grains. Additionally, the physical orientations of the phases with respect to each other could be a primary controlling the ground boundary morphologies. 


\section{References:}

[1] J. Boesenberg, J. Delaney, and R. Hewins, Geochimica et Cosmochimica Acta 89 (2012), p. 134.

[2] E. Scott, Lunar and Planetary Science Conference XXXVIII (2007), \#2284.

[3] L. Garvie, Meteoritical Bulletin 106 (2018), in prep.

[4] J. Boesenberg et al, Lunar and Planetary Science Conference XLIX (2018), \#1556.

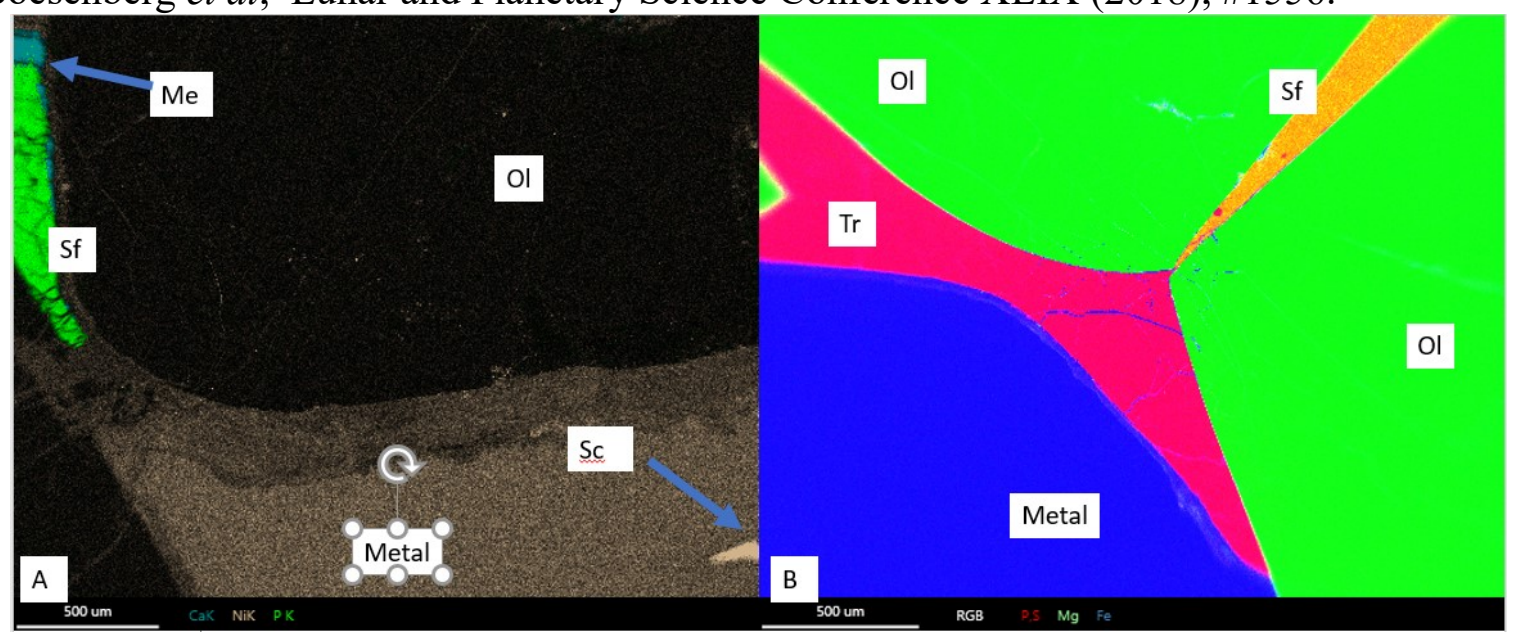

Figure 1. (A) Merrilite (Me) was found next to stanfieldite (Sf), while schreiberstite (Sc) was found inside the metal in a mixed elemental map $($ Green $=\mathrm{K}, \mathrm{Blue}=\mathrm{Ca}$, and Light Orange $=\mathrm{Ni})$. (B) In this phase map, the olivine $(\mathrm{Ol})$ boundary with standfieldite is straight, while the boundary with troilite $(\mathrm{Tr})$ is rounded in nature.

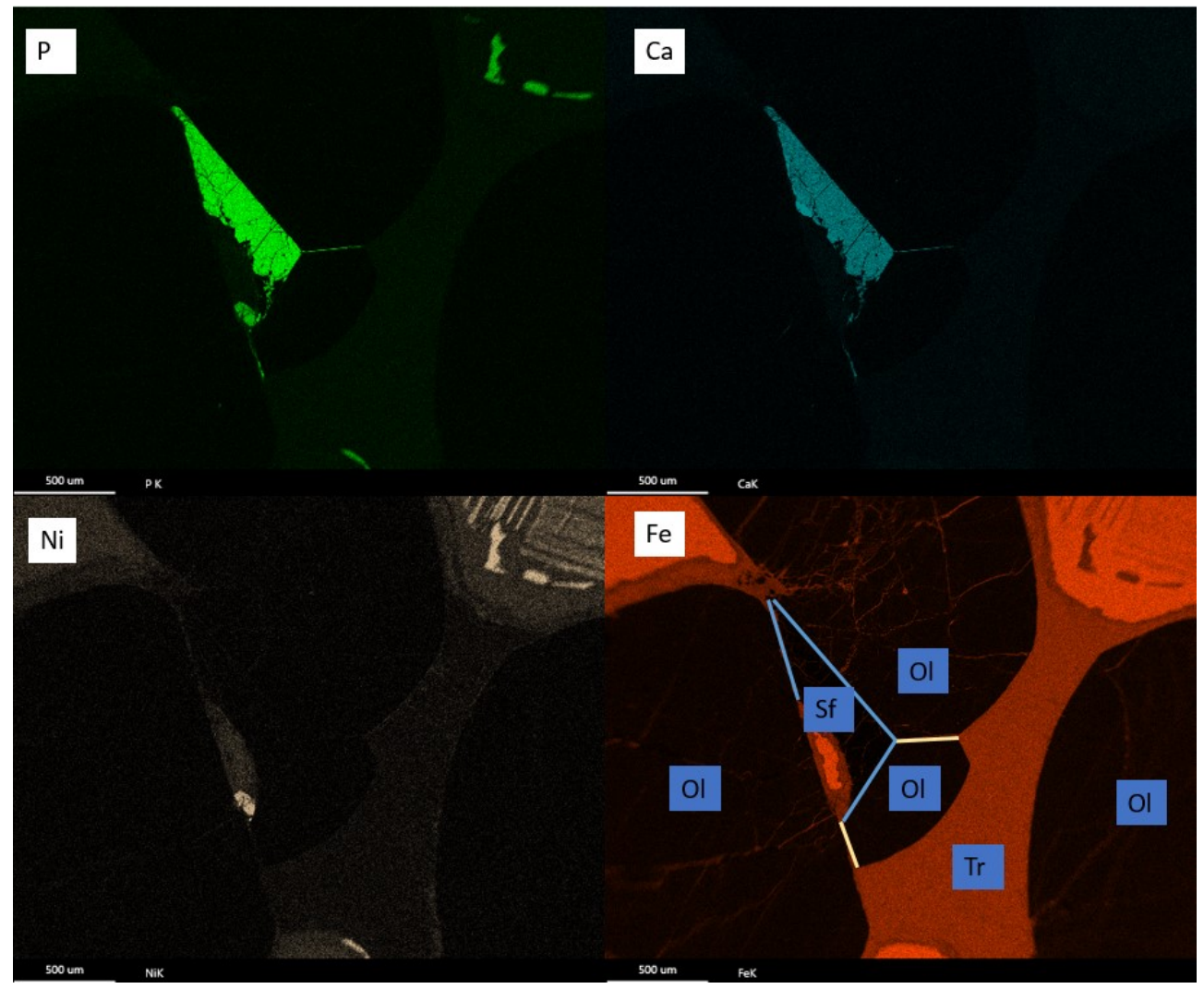

Figure 2. These elemental maps show the presence of a large stanfieldite grain bounded by olivines, with a clear triple junction present. The olivine-troilite phase boundary is again rounded in nature. 